\title{
Mechano-Optical Switching of a Single Molecule with Doublet Emission
}

\author{
Jiří Doležal ${ }^{1,2}$, Pingo Mutombo ${ }^{1,3}$, Dana Nachtigallová ${ }^{4,5}$, Pavel Jelínek ${ }^{1,5}$, Pablo Merino ${ }^{6,7 *}$, \\ Martin Švec ${ }^{1,5 *}$ \\ ${ }^{1}$ Institute of Physics, Czech Academy of Sciences, Cukrovarnická 10/112, CZ16200 Praha 6, Czech Republic \\ ${ }^{2}$ Faculty of Mathematics and Physics, Charles University, Ke Karlovu 3, CZ12116 Praha 2, Czech Republic \\ ${ }^{3}$ Department of Petrochemistry and Refining, University of Kinshasa, Kinshasa, Democratic Republic of Congo \\ ${ }^{4}$ Institute of Organic Chemistry and Biochemistry of the Czech Academy of Sciences, Flemingovo náměstí 542/2 \\ CZ16000, Praha 6, Czech Republic \\ ${ }^{5}$ Regional Centre of Advanced Technologies and Materials, Šlechtitelů 27, CZ78371 Olomouc, Czech Republic \\ ${ }^{6}$ Instituto de Ciencia de Materiales de Madrid, CSIC, Sor Juana Inés de la Cruz 3, E28049 Madrid, Spain \\ ${ }^{7}$ Instituto de Física Fundamental, CSIC, Serrano 121, E28006 Madrid, Spain \\ *corresponding authors: pablo.merino@csic.es, svec@fzu.cz
}

1. AFM simulations of $\mathrm{NaCl}$ and adsorbed $\mathrm{CuPc}$ molecule.

2. AFM images of the steady and dynamic configurations.

3. Statistical analysis of the exciton switching on $2 \mathrm{ML}$ and $3 \mathrm{ML}$ of $\mathrm{NaCl}$.

4. Switching steady and dynamic state.

5. Kelvin probe measurement on steady and dynamic states.

6. Theoretical stability of steady and dynamic states.

7. 2D representation of the Hartree potential for the dynamic and steady states

8. Exciton energy calculations 


\section{AFM simulations of $\mathrm{NaCl}$ and adsorbed $\mathrm{CuPc}$ molecule.}
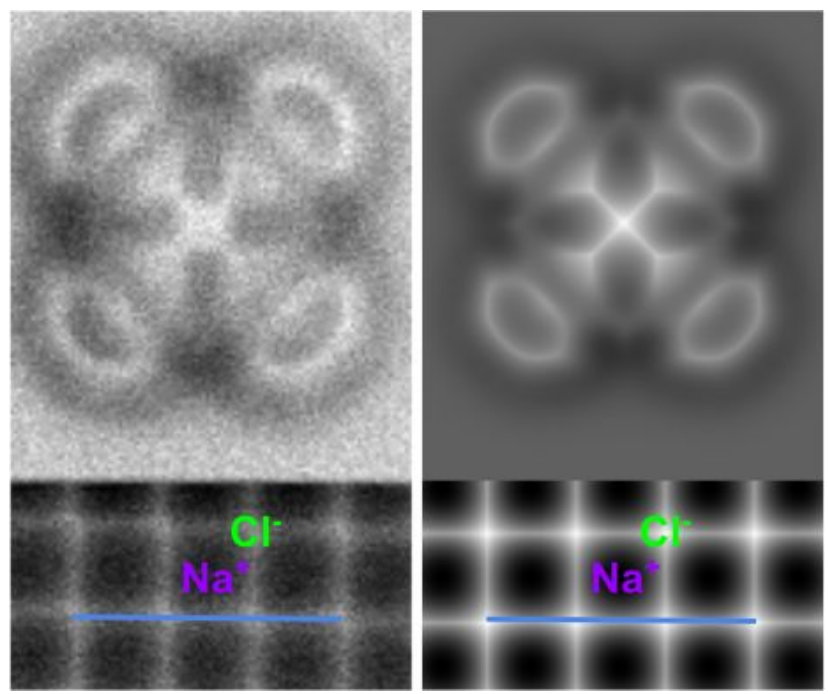

Figure S1: Comparison of the AFM frequency shift: experimental data (left) and theoretical simulation (right) of $\mathrm{CuPc}$ on $2 \mathrm{ML}-\mathrm{NaCl}$ in the steady configuration, scanned with a CO-functionalized tip. The images of the $\mathrm{NaCl}$ lattice (bottom parts) are obtained with $300 \mathrm{pm}$ smaller tip-sample distance with respect to the CuPc region. The image sizes are $1.70 \times 2.95 \mathrm{~nm}^{2}$. Bars (light blue) of $3 \mathrm{NaCl}$ unit cell lengths are added for size comparison. Simulated AFM images were obtained using the ProbeParticle code ${ }^{1,2}$, which takes into account the van der Waals (vdW) and the electrostatic interactions between the surface and the CO tip. All the simulations were done with a charge scaling of $-0.5 e^{-}$a lateral stiffness of $0.25 \mathrm{~N} / \mathrm{m}$. The Hartree potential calculated from the DFT calculations was used to obtain the electrostatic forces. 


\section{AFM images of the steady and dynamic configurations.}

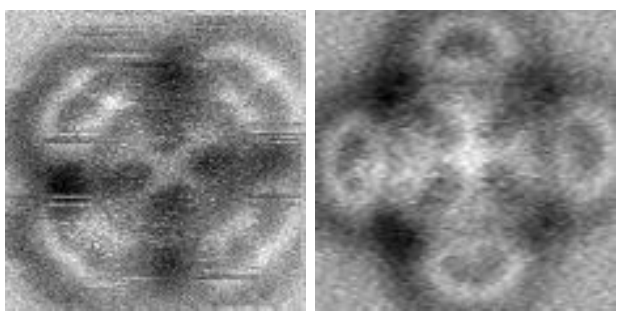

Figure S2: Comparison of the AFM images of the dynamic (left) and steady (right) states on 2 $M L$ of $\mathrm{NaCl}$ on $\mathrm{Ag}(111)$, scanned with a CO-functionalized tip. In the dynamic image the fast rotational switching of the CuPc leads to a double appearance of the peripheral benzene rings, as a result of temporal averaging of the AFM signal. The fourfold symmetry of the dynamic configuration indicates that the molecule stays on the same $\mathrm{Cl}^{-}$ion after its rotation. Image parameters: $1.6 \times 1.6 \mathrm{~nm}^{2}, \Delta f_{\min }=-9.5 \mathrm{~Hz}, \Delta f_{\max }=2.6 \mathrm{~Hz}, 5 \mathrm{mV}$ setpoint. 
3. Statistical analysis of the exciton switching on $2 \mathrm{ML}$ and $3 \mathrm{ML}$ of $\mathrm{NaCl}$.

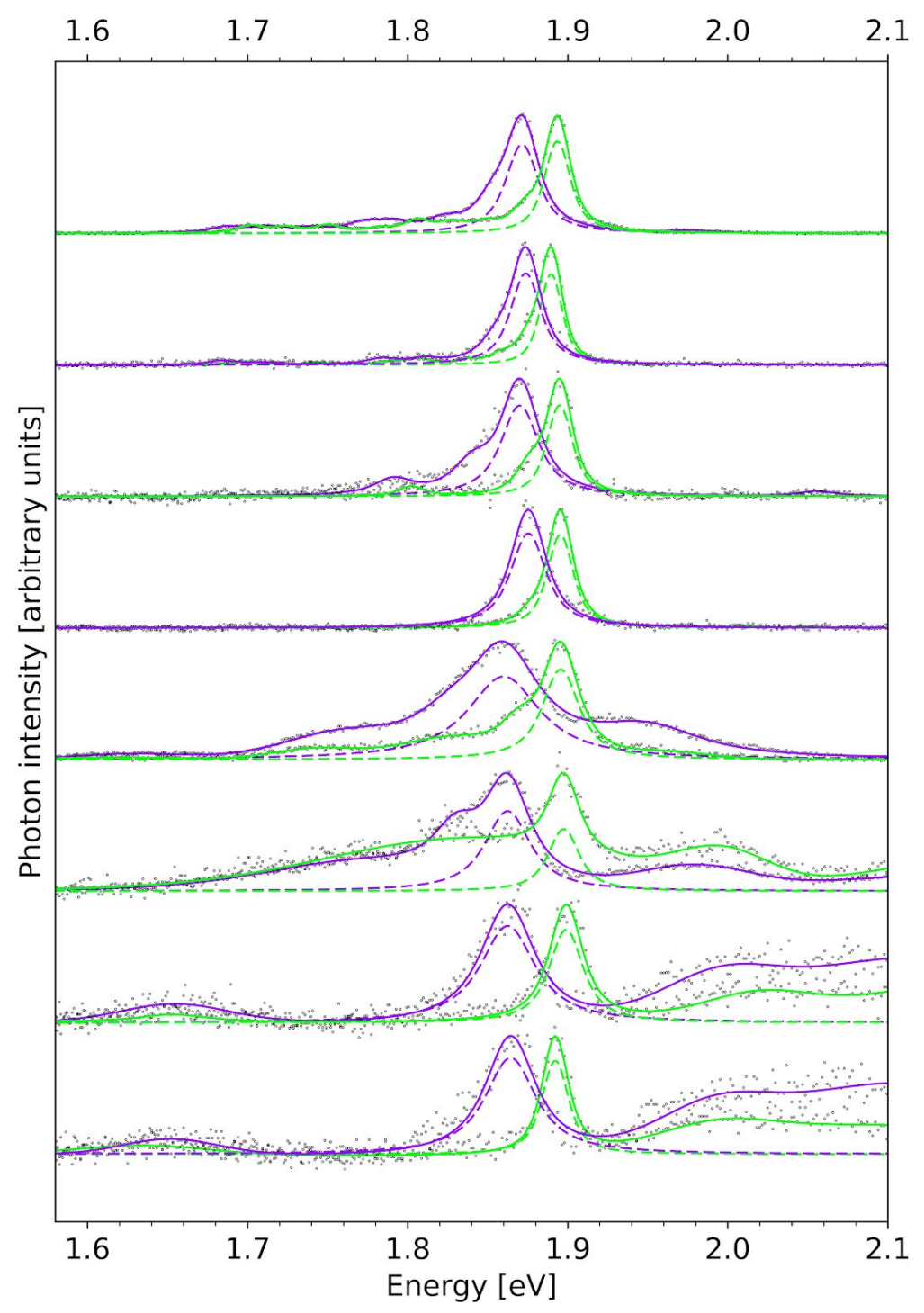

Figure S3: Overview and fit of STML spectra obtained on CuPC molecules before (green) and after stabilization (violet). Spectra are normalized to their maxima. Main fitted components corresponding to the $D_{1}-D_{0}$ transitions (dashed lines) are rescaled to $80 \%$ of their original amplitude for clarity. The measurements were performed for 4 molecules on $2 \mathrm{ML}-\mathrm{NaCl}$ (lower half of the plot) and for 4 molecules on $3 \mathrm{ML}$ (upper half of the plot). For fitting of all excitonic components, pseudo Voigt profiles were used with a gaussian full width at half maximum fixed to $10 \mathrm{meV}$, equivalent to $3.5 \mathrm{~nm}$ spectral resolution at $650 \mathrm{~nm}(1.907 \mathrm{eV})$. Spectra were fitted using the program KolXPD. ${ }^{3}$ 


\begin{tabular}{|c|c|c|c|c|c|c|c|c|}
\hline & & \multirow{2}{*}{\multicolumn{2}{|c|}{$\begin{array}{l}\text { Steady CuPc } \\
E_{\text {OPT }}{ }^{N a}[e V] f_{L}{ }^{N a}[m e V]\end{array}$}} & \multirow{2}{*}{\multicolumn{2}{|c|}{$\begin{array}{l}\text { Dynamic CuPc } \\
\mathrm{E}_{\mathrm{OPT}}{ }^{\mathrm{Cl}}[\mathrm{eV}] \mathrm{f}_{\mathrm{L}}^{\mathrm{Cl}}[\mathrm{meV}]\end{array}$}} & \multirow{2}{*}{\begin{tabular}{|l|} 
Difference \\
$\mathrm{E}_{\mathrm{OPT}}{ }^{\mathrm{Cl}}-\mathrm{E}_{\mathrm{OPT}}{ }^{\mathrm{Na}}[\mathrm{meV}]$
\end{tabular}} & \multicolumn{2}{|c|}{ Avg. broadening } \\
\hline & & & & & & & $\mathrm{f}_{\mathrm{L}}^{\mathrm{Na}} / \mathrm{f}_{\mathrm{L}} \mathrm{Cl}$ & $\%$ \\
\hline \multirow[t]{4}{*}{$3 \mathrm{ML} \mathrm{NaCl}$} & average & 1.873 & 20.0 & 1.893 & 13.1 & 20.6 & 1.63 & 63 \\
\hline & st. deviation & 0.002 & 2.6 & 0.002 & 1.5 & 3.4 & - & - \\
\hline & $\max$ & 1.876 & 24.0 & 1.895 & 14.1 & 25.0 & 1.74 & 74 \\
\hline & $\min$ & 1.870 & 16.4 & 1.890 & 10.4 & 15.6 & 1.42 & 42 \\
\hline \multirow[t]{4}{*}{$2 \mathrm{ML} \mathrm{NaCl}$} & average & 1.862 & 38.4 & 1.896 & 19.4 & 34.0 & 2.03 & 103 \\
\hline & st. deviation & 0.002 & 8.7 & 0.003 & 3.3 & 3.5 & - & - \\
\hline & $\max$ & 1.864 & 52.0 & 1.899 & 23.6 & 37.0 & 2.41 & 141 \\
\hline & $\min$ & 1.860 & 28.0 & 1.892 & 14.5 & 28.0 & 1.67 & 67 \\
\hline
\end{tabular}

Table S1: Fitting and statistical parameters of optical spectra acquired on the steady and dynamic configurations of CuPc on $2 \mathrm{ML}$ and $3 \mathrm{ML}-\mathrm{NaCl}$. The parameters $\mathrm{E}_{\mathrm{OPT}}^{\mathrm{Na}, \mathrm{Cl}}$ and $f_{L}$ denote the energy position of the line and the full width at half maximum of the Lorentzian component of the Voigt profile used for the fitting, respectively. 
4. Switching steady and dynamic state.
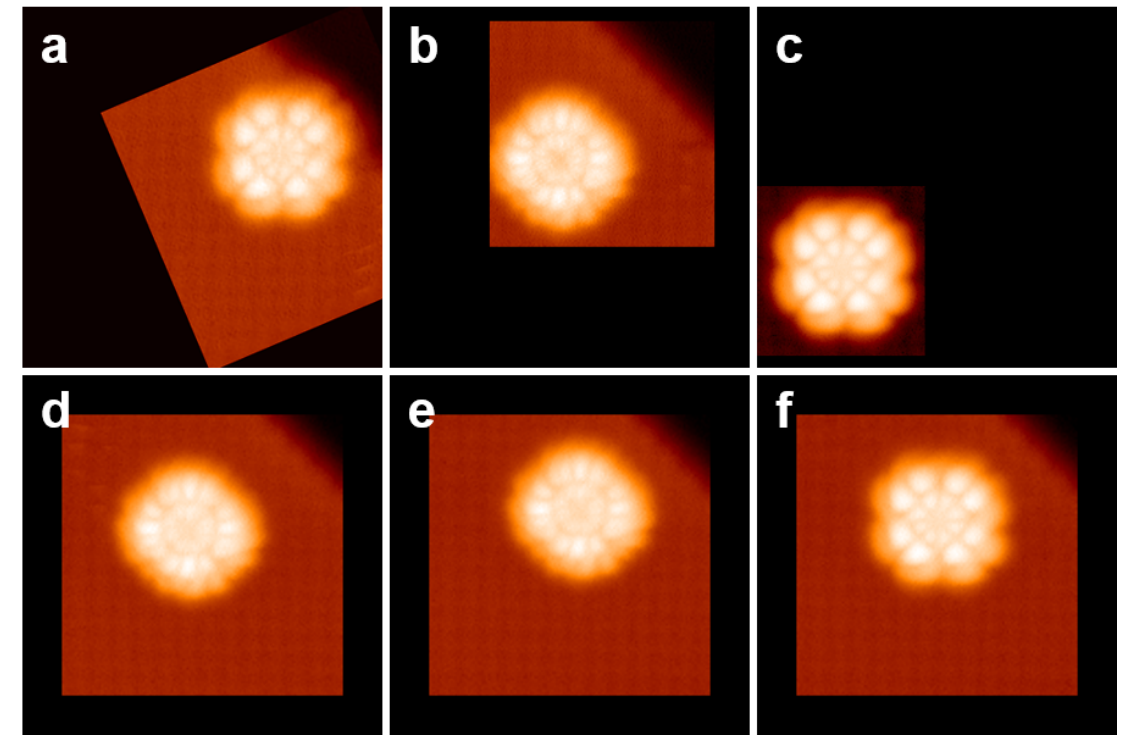

Figure S4: (a-f) Series of constant current STM images taken with a CO-tip (-2.6 V, 1 pA) showing the same CuPc molecule on a $3 \mathrm{ML}-\mathrm{NaCl}$ island being switched back and forth between dynamic and steady states by atomic scale voltage-induced and lateral-force manipulation. Translation, scale and rotation are applied to each image to achieve a correct position in a common reference frame of $7 \times 7 \mathrm{~nm}^{2}$, according to their recorded scanning parameters. 


\section{Kelvin probe measurement on steady and dynamic states.}

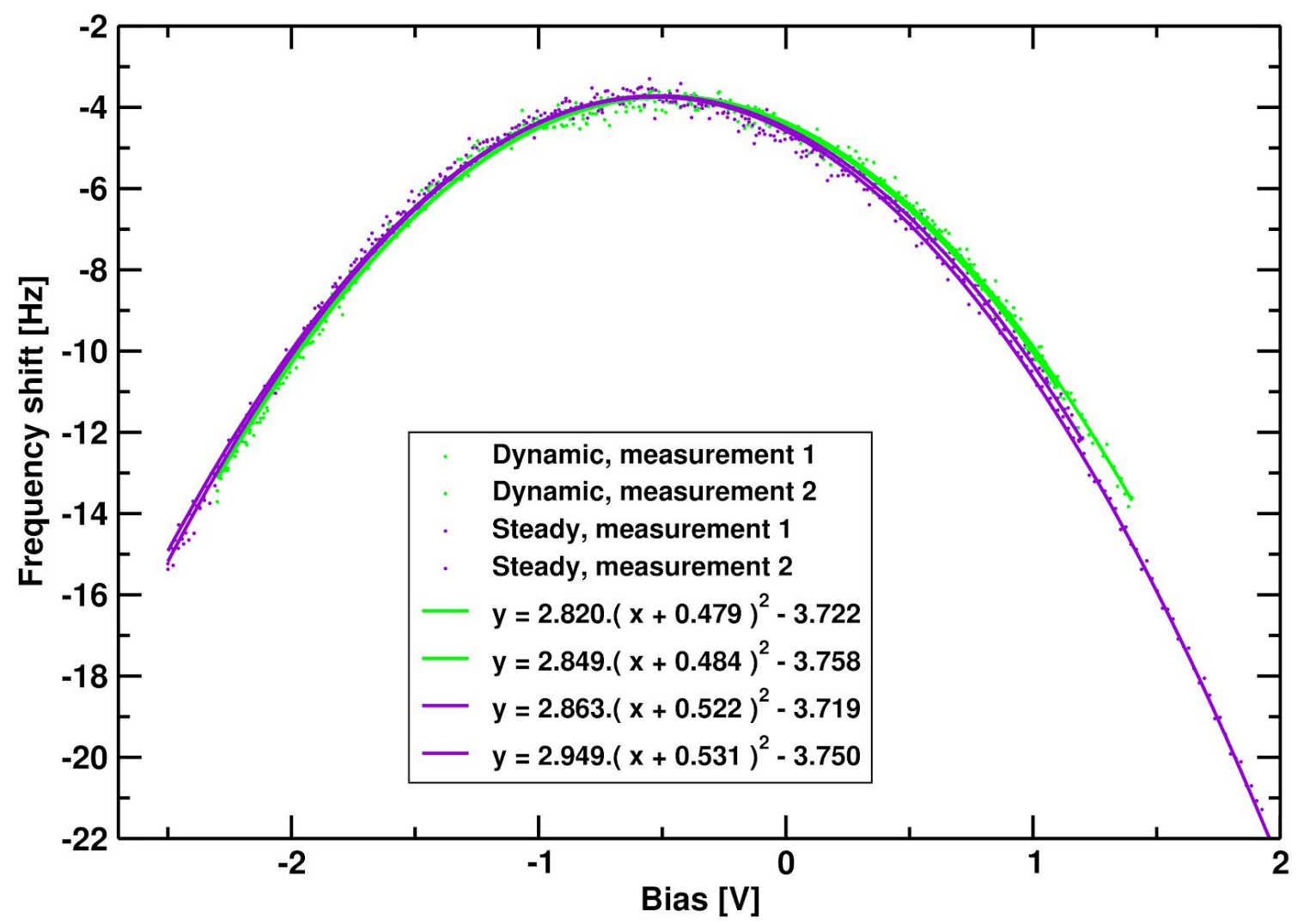

Figure S5: Kelvin probe measurements on the CuPc / $3 \mathrm{ML}-\mathrm{NaCl}$, switched from dynamic to steady configurations, taken at the molecular lobe position. The data were evaluated using a parabolic fit. The local potential difference on the steady configuration is shifted by $-45 \mathrm{meV}$ with respect to the dynamic configuration. 
6. Theoretical stability of steady and dynamic states.

\begin{tabular}{|r|r|r|}
\hline $\begin{array}{l}\text { CuPc orientation to } \\
\mathrm{NaCl}[100]\end{array}$ & on top of $\mathrm{Na}^{+}[\mathrm{eV}]$ & on top of $\mathrm{Cl}^{-}[\mathrm{eV}]$ \\
\hline $0^{\circ}$ & 0.58 & 0.09 \\
\hline $15^{\circ}$ & 0.49 & $0.11($ rotates to \\
$\left.9^{\circ}\right)$ \\
\hline $30^{\circ}$ & rotates toward & rotates toward \\
$45^{\circ}$ & $15^{\circ}$ \\
\hline $45^{\circ}$ & 0.000 & 0.46 \\
\hline
\end{tabular}

Table S2: Comparison of the total energy offsets, calculated for various adsorption configurations of CuPc on $2 \mathrm{ML}-\mathrm{NaCl}(100)$. The steady configuration (on top of the $\mathrm{Na}$ ion, $45^{\circ}$ with respect to the [100] $\mathrm{NaCl}$ axis) with the minimum total energy is taken as a reference. 
7. 2D representation of the Hartree potential for the dynamic and steady states.
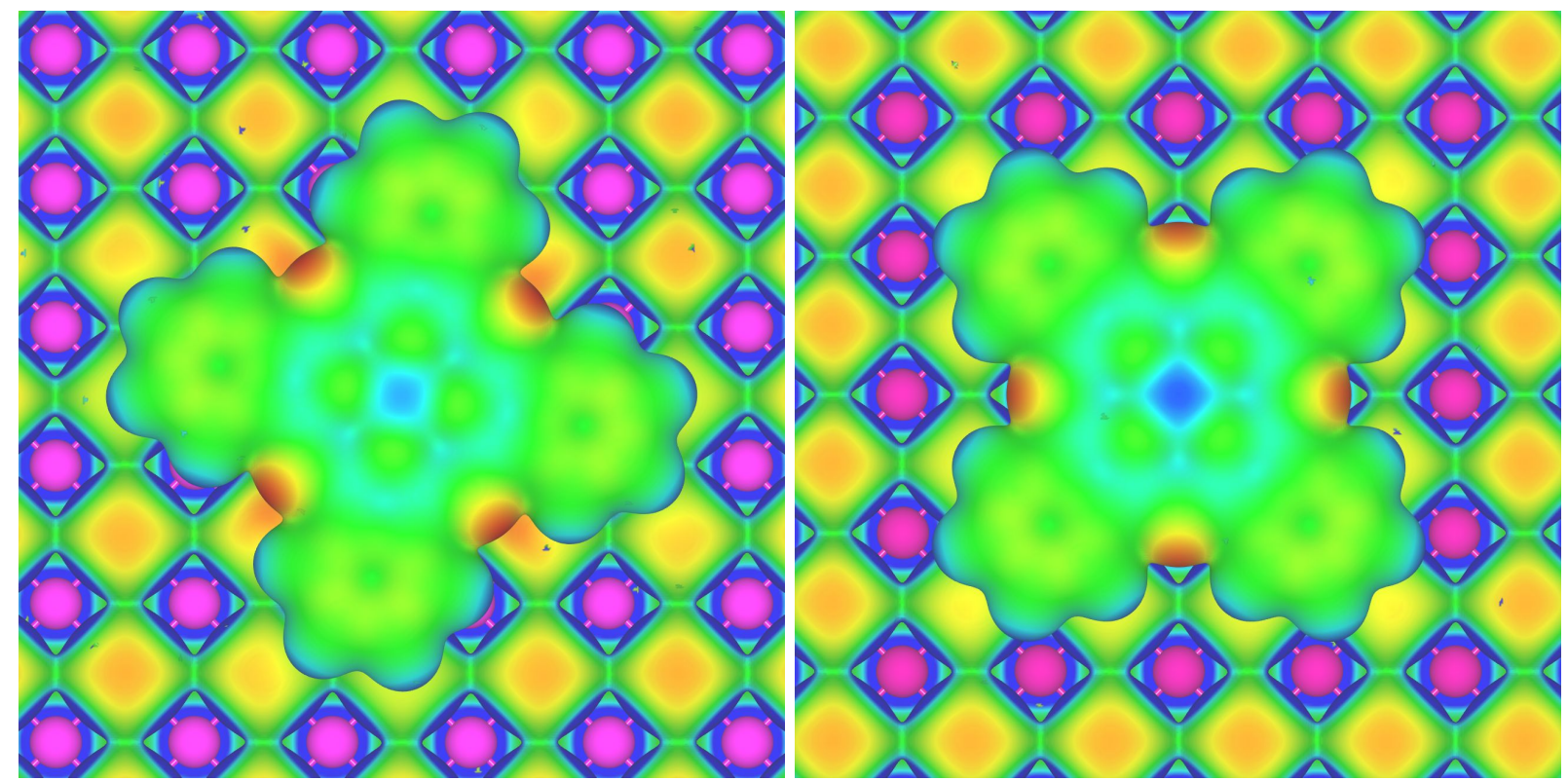

Figure S6: Calculated Hartree potential of $\mathrm{CuPC}$ on $\mathrm{NaCl}$ for the steady and dynamic configurations (metal core on $\mathrm{Na}$ and $\mathrm{Cl}$ atoms respectively) obtained from the DFT calculations, projected onto the isosurface of constant total charge density $0.025 \mathrm{e}^{-} / \mathrm{A}^{3}$. Low potential values correspond to blue color and high potential values to red color, respectively. Minimum and maximum values are identical for both panels. The Hartree potentials reveal intramolecular charge transfer between the pyrrole rings and imine nitrogens. 


\section{Exciton energy calculations}

\begin{tabular}{|c|c|c|c|c|c|c|c|}
\hline \multirow[b]{2}{*}{ method } & \multirow[b]{2}{*}{ configuration } & \multicolumn{3}{|c|}{ unrelaxed transition } & \multicolumn{3}{|c|}{ geometrically optimized } \\
\hline & & $\begin{array}{c}\text { spin up } \\
(\uparrow)\end{array}$ & $\begin{array}{c}\text { spin down } \\
(\downarrow)\end{array}$ & $E_{O P T} \downarrow-E_{O P T} \uparrow$ & $\begin{array}{c}\text { spin up } \\
(\uparrow)\end{array}$ & $\begin{array}{c}\text { spin down } \\
(\downarrow)\end{array}$ & $E_{O P T} \downarrow-E_{O P T} \uparrow$ \\
\hline LC-wHPBE & isolated & 1.968 & 2.009 & 0.041 & 1.693 & 1.734 & 0.041 \\
\hline wB97XD & isolated & 1.823 & 1.86 & 0.037 & 1.65 & 1.686 & 0.036 \\
\hline \multirow{3}{*}{ LC-wHPBE } & on $\mathrm{Na}$ & 1.961 & 2.002 & 0.041 & - & - & - \\
\hline & on $\mathrm{Cl}$ & 1.983 & 2.024 & 0.041 & - & - & - \\
\hline & $E_{O P T}^{C l}-E_{O P T}{ }^{N a}$ & 0.022 & 0.022 & & & & \\
\hline \multirow{3}{*}{ wB97XD } & on $\mathrm{Na}$ & 1.811 & 1.848 & 0.037 & - & - & - \\
\hline & on $\mathrm{Cl}$ & $1.855^{*}$ & 1.864 & $0.009^{*}$ & - & - & - \\
\hline & $E_{O P T}^{C l}-E_{O P T}{ }^{N a}$ & $0.044^{*}$ & 0.022 & & & & \\
\hline
\end{tabular}

Table S3: Summary of the unrelaxed and geometrically optimized exciton energies for the isolated CuPc and CuPc subjected to the field of $2 \mathrm{ML}-\mathrm{NaCl}$, represented by point charges, with geometries corresponding to the steady and dynamic configurations. The indices of the exciton energies $\left(E_{\mathrm{OPT}}\right)$ denote the adsorption site/configuration ( $\mathrm{Na}$ - steady, $\mathrm{Cl}$ - dynamic). The arrows mark the spin subsystem in which the electron has been transferred from HOMO to LUMO. The energies marked by asterisk (*) were obtained from the last step before the simulated system relaxed to its ground-state $\left(D_{0}\right)$ orbital occupancy, due to instability of the wB97XD simulation method in the case of the dynamic configuration, with excitation within the spin up subsystem. The differences $E_{O P T} \downarrow-E_{O P T} \uparrow$ are given to demonstrate independence of the result among the used methods and configurations, which allows a reliable estimate of the difference in the exciton energies $E_{O P T}^{C l}-E_{O P T}{ }^{N a}$. All energies are given in $\mathrm{eV}$. 


\section{Supplementary references}

1. Hapala, P.; Temirov, R.; Tautz, F. S.; Jelínek, P. Origin of High-Resolution IETS-STM Images of Organic Molecules with Functionalized Tips. Phys. Rev. Lett. 2014, 113, 226101.

2. Hapala, P.; Kichin, G.; Wagner, Ch.; Tautz, F. S.; Temirov, R.; Jelínek, P. Mechanism of High-Resolution STM/AFM Imaging with Functionalized Tips. Physical Review B 2014, 90, 085421.

3. Libra J. KoIXPD, 1.8.0; kolibrik.net: Czech Republic, 2020. 